\title{
DE LA PLANEACIÓN A LA GESTIÓN AMBIENTAL COLECTIVA: POSIBILIDADES EN BOGOTÁ-COLOMBIA
}

\section{FROM PLANNING TO A COLLECTIVE ENVIRONMENTAL MANAGEMENT: POSSIBILITIES IN BOGOTÁ-COLOMBIA}

\author{
Adriana Posada ${ }^{1}$ \\ ${ }^{1}$ Economista Agrícola, Esp. Gestión Social y Ambiental. M.Sc. Planeación Urbana y Regional, docente Facultad Ingeniería \\ Geográfica y Ambiental, Universidad de Ciencias Aplicadas y Ambientales U.D.C.A, calle 222 \#55-37, Bogotá, D.C., Colom- \\ bia, e-mail: aposada@udca.edu.co; adriaposada@yahoo.es
}

Rev. U.D.CA Act. \& Div. Cient. 17(1): 257-264, Enero-Junio, 2014

\section{RESUMEN}

Bogotá ha avanzado en el manejo ambiental de su territorio, pero el camino para hacer realidad un mejor entorno ambiental apenas empieza y, para ello, se hace necesario pasar de la planeación a la gestión ambiental colectiva. Se requiere reflexionar y alertar sobre la necesidad urgente de utilizar la cantidad de instrumentos existentes para incidir con eficiencia en el mejoramiento de la calidad de vida. El Distrito Capital está generando una diferencia en el enfoque de la planeación ambiental, pero falta, por una parte, el cambio de mentalidad colectiva para apropiarse del futuro y, por otra, importantes y reales esfuerzos públicos que muestren la materialización de las acciones, en la relación sociedad naturaleza. En suma, sin la gestión ambiental colectiva, la planeación carece de sentido, pues el primer aliento de vida de la planeación lo inyectan las comunidades al plasmar sus sueños en un ideal que, luego, podrán ayudar a hacerlo real, a través de la gestión participativa.

Palabras clave: Conflicto socio ambiental, gestión territorial, planeación territorial.

\section{SUMMARY}

Bogota has advanced in the environmental management of its territory, but the purpose to create a better surrounding just begins and for this it is necessary to move from the planning to the collective environmental management. Reflect and highlight the urgent need to use the amount of existing instruments to influence effectively in improving the quality of life is required. The District Capital is generating a difference in approach to environmental planning; but missis on one hand, to appropriate future collective mentality change, and for another, actual and important public efforts that show the materialization of the actions in the relationship nature- society. In short, without the collective environmental management planning, planning is meaningless, because its first breath of life is injected by the communities, capturing their dreams in an ideal which can then help to make it real through participatory management.

Key words: Socio-environmental conflict, land management and territorial planning.

\section{INTRODUCCIÓN}

En Colombia han pasado 23 años desde que la Constitución Política incluyó, en el Artículo 58, el derecho a gozar de un ambiente sano (REPÚBLICA DE COLOMBIA, 1991); a partir de ello, los entes territoriales han tenido la decisión de circunscribir planes de ordenamiento ambiental de su territorio y presupuestos, para garantizar las acciones necesarias que conduzcan a la recuperación ambiental, con el fin de lograr una mejor calidad de vida; sin embargo, la necesidad de mejorar las condiciones ambientales del territorio, data de muchos más años antes que la norma lo determinara. La norma no es la que le dio el origen a la necesidad de mejorar el ambiente, todo lo contrario, las dificultades antrópicas generadas por un degradado entorno natural son el punto de partida para llevar a las políticas y a la normativa del país y del mundo a pensar en instrumentos para solucionar, en parte, el problema ambiental.

Más de dos décadas después de que en Colombia se hubiesen promulgado diferentes políticas, normas, planes y programas, no concretamos aún las acciones, debido a que damos vueltas en círculo sobre el qué hacer, el cómo, el dónde, el con qué, pero no llevamos con efectividad a la práctica, los distintos instrumentos creados. Ello, debido, en gran parte, 
a que no hemos podido pasar de la fragmentación de las acciones, a la visión sistémica y, mucho menos, a las acciones concretas en la relación sociedad naturaleza.

El país creó un sistema de planeación complejo, con instancias diversas, multiplicidad de instrumentos, actores y niveles, con ámbitos de competencias medianamente determinadas e, incluso, donde confluyen con entidades e instituciones de otra naturaleza (Quijano, 2012).

La urgencia de hacer de Bogotá un territorio más armónico se destaca no solamente por ser la capital colombiana, sino por contar con ventajas comparativas en su ubicación, en la conformación de su estructura natural, en la responsabilidad de y para más de siete millones de personas y en la administración de recursos financieros, que se deben invertir en el mejoramiento de las condiciones actuales.

Preguntémonos si en el territorio bogotano se evidencian cambios notorios positivos en su estructura natural, ċcon qué instrumentos se cuenta para hacerlo posible?, cंson efectivos en el ámbito natural y social?, cंquiénes son los responsables de hacer el cambio?, ċlas personas del común conocemos los problemas, participamos en su solución y hacemos posible algún cambio? Es entonces necesario indagar por los instrumentos que se están utilizando para lograr tal armonía y sobre todo, se requiere establecer si hacen parte de un proceso socio ambiental articulado, integral y colectivo.

Por ello, como lo plantea Retoloza (2010), al vivir en tiempos complejos y por momentos conflictivos, necesitamos disponer de instrumentos más flexibles, que nos permitan planificar y monitorear nuestras acciones en contextos inciertos, emergentes y complejos; siempre desde una lógica flexible, no rígida.

Fernández (2000) destaca la diferencia entre el proceso técnico de la planificación y el de la administración del territorio; este último, como el más complejo, en tanto debe efectivizar los planes, a través de diversas clases de controles, monitoreos o acciones instrumentales que, muy a pesar de su existencia, han creado una creciente desconfianza por su inoperatividad y por la dificultad de implementarlos.

En teoría, según la Alcaldía Mayor de Bogotá -AMB- (2008), el Distrito quiere enmarcar y articular las acciones de todos los actores estratégicos hacia el fortalecimiento de la gestión ambiental del Distrito Capital y de la región, asumiendo un proceso en el que intervienen diferentes actores estratégicos, a través de la formulación y adopción de políticas públicas.

Respecto a lo anterior, Velásquez (2010) aplaude los aciertos en materia de planificación territorial que ha tenido el distrito, pero insiste en que falta superar problemas relativos a la práctica de la planificación participativa, entre ellos, el escaso interés de los actores políticos en el proceso.

Como un importante avance conceptual, la AMB (2010) asume explícitamente el concepto de gestión ambiental territorial e introduce tres elementos principales para conocerla: la construcción social del territorio, la planificación participativa y la estructuración de la inversión ambiental local y territorial.

Por ello, el objetivo de este escrito es aportar elementos para comprender que los conflictos ambientales como parte de la dinámica del sistema territorial, necesitan no sólo planificar acciones para solucionarlos, sino establecer, con mucha fuerza, la integración de diferentes actores transformadores, para ejecutar con mayor acierto lo planeado.

\section{MATERIALES Y MÉTODOS}

El Distrito de Bogotá reconoce diferentes territorios de interés ambiental y ha avanzado en la planificación y en la gestión que le compete, según su contexto particular (Figura 1). Entonces, un análisis de planeación y de gestión ambiental del Distrito, incluye, no solamente la mirada endógena, sino elementos exógenos, propios de su estructura ecológica principal y de su connotación como capital colombiana.

Para analizar la información, se utilizan los insumos que permiten obtener una mirada comparativa, no sólo del uso y devenir de los instrumentos de planeación y de gestión, sino de las responsabilidades y las posibilidades que tiene el Distrito Bogotá para recuperar gradualmente y en asocio con la sociedad, los elementos básicos del tejido natural. Tal información se subdivide en:

1. El uso de los conceptos y su significancia: Se utilizaron consideraciones de autores que han disertado sobre la planificación y la gestión ambiental; se analizaron las principales políticas relacionadas con el tema y se realizaron una docena de entrevistas individuales, a personas claves en los procesos de planificación y de gestión ambiental territorial de Bogotá.

\section{El uso de los instrumentos de planeación y de gestión y} su correspondiente evolución: Se analizaron los instrumentos de planeación y de gestión con que cuenta el Distrito, comparando los cambios entre cuatro periodos de gobierno $\mathrm{y}$ analizando el enfoque de las diferentes propuestas consignadas en las fichas de inversión distrital.

3. La comparación entre variables claves de los principales entes territoriales en Colombia: Para los seis principales municipios de Colombia (Bogotá, Medellín, Barranquilla, Cali, Cartagena y Santa Marta), se comparan las cifras de población total, área en $\mathrm{km}^{2}$ y presupuesto de inversión para la vigencia actual, según los datos del DNP (2013). 


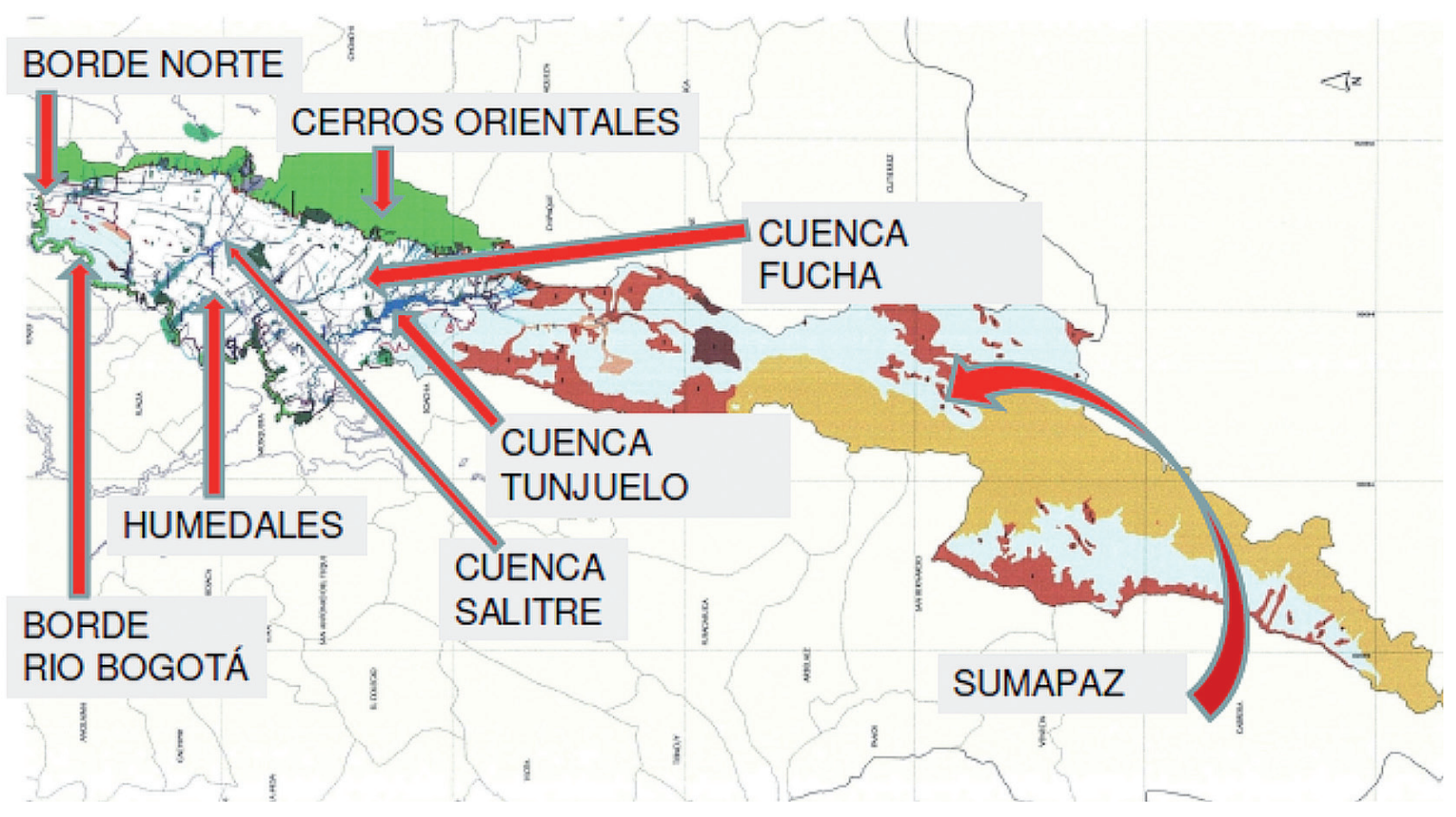

Figura 1. Ubicación de los territorios de interés ambiental reconocidos por el Distrito Bogotá. AMB (2010).

\section{RESULTADOS Y DISCUSIÓN}

Los instrumentos de planeación con que cuenta Bogotá: Desde el ámbito nacional, el MAVDT (2008) establece el marco institucional para la gestión ambiental, de acuerdo a lo previsto en la Ley 99 de 1993, que define las siguientes correspondencias de planeación y de gestión ambiental territorial así:

- El MAVDT, como cabeza del Sistema Nacional Ambiental (SINA) y como rector de la política ambiental.

- Las Corporaciones Autónomas Regionales y las de Desarrollo Sostenible, como autoridades ambientales en las áreas urbanas y rurales de los municipios de su jurisdicción.

- Las autoridades ambientales al interior del perímetro urbano de los grandes centros urbanos.

Desde el ámbito distrital, Bogotá ha contado con cuatro planes de gestión ambiental: el primero, en 1992 (inclusive, antes de la promulgación de la Ley 99 de 1993, que crea al MMA); luego, en el siguiente periodo de gobierno, en 1995; posteriormente, el tercero, se adoptó mediante el Decreto 061 de 2003, con un alcance de nueve años (2001-2009); más adelante, el Decreto Distrital 456 de 2008, permitió adoptar el actual Plan de Gestión Ambiental, con una vigencia de largo plazo de 30 años (2008-2038).

Como consecuencia, la AMB (2010) asume el enfoque territorial de la gestión ambiental reconociendo en su política sectorial ambiental, las dinámicas de organización de los territorios. Además, existen otros insumos que dan luces sobre la evolución del concepto y de los instrumentos utilizados para la recuperación ambiental territorial en Bogotá. Como se observa en el cuadro 1, se trata, por una parte, de los denominados "instrumentos de planificación y de gestión" y, por otra, de los nombrados como "instrumentos de planeación ambiental".

Para completar lo anterior, existen un SIGA -Sistema de Indicadores de Gestión Ambiental del Distrito Capital- y un Plan de Gestión para el Desarrollo Rural Sostenible, en el cual, se destaca la estrategia denominada "gestión social integral del territorio", que tiene por objetivo acompañar y promover procesos de gestión social de la base natural y de los territorios, fortaleciendo la capacidad de negociación de los actores locales y, específicamente, de los productores rurales (AMB, 2009).

Respecto a la corroboración de los proyectos específicos sobre gestión ambiental en el territorio Bogotano, se destacan los siguientes cuatro proyectos (el 30\%), entre los 12 aprobados a la fecha, para la vigencia 2012-2016:

- Proyecto 131. Participación ciudadana y educación ambiental como instrumentos de gestión para la apropiación social de los territorios ambientales.

- Proyecto 817. Planeación ambiental participativa, comunicación estratégica y fortalecimiento de procesos de for- 
Cuadro 1. Síntesis de los instrumentos de planificación y de gestión ambiental utilizados en Bogotá.

\begin{tabular}{|c|c|c|c|}
\hline & Locales & Territoriales & Distritales \\
\hline $\begin{array}{l}\text { Instrumentos de planifi- } \\
\text { cación y de gestión. }\end{array}$ & $\begin{array}{l}\text { Agendas ambientales } \\
\text { locales; planes ambi- } \\
\text { entales locales; plan de } \\
\text { desarrollo local. }\end{array}$ & $\begin{array}{l}\text { Sistema planificación distrital; políticas } \\
\text { ambientales de humedales, de rurali- } \\
\text { dad, de reasentamientos, de preven- } \\
\text { ción y manejo de riesgo, de salud y am- } \\
\text { biente; planes de desarrollo, de gestión } \\
\text { ambiental, de ordenación y manejo de } \\
\text { ecosistemas, de ordenamiento territo- } \\
\text { rial, de desarrollo local, de control de } \\
\text { los vertimientos. }\end{array}$ & $\begin{array}{l}\text { Plan de gestión ambien- } \\
\text { tal distrital; plan de or- } \\
\text { denamiento territorial; } \\
\text { planes maestros; plan de } \\
\text { desarrollo distrital; siste- } \\
\text { ma interinstitucional. }\end{array}$ \\
\hline $\begin{array}{l}\text { Instrumentos de pla- } \\
\text { neación ambiental. }\end{array}$ & $\begin{array}{l}\text { Plan de acción cuatrien- } \\
\text { al Ambiental; planes In- } \\
\text { stitucionales de gestión } \\
\text { ambiental. }\end{array}$ & & $\begin{array}{l}\text { Planes de ordenación y } \\
\text { manejo de cuencas hi- } \\
\text { drográficas; planes de } \\
\text { manejo ambiental de áre- } \\
\text { as de interés ambiental. }\end{array}$ \\
\hline
\end{tabular}

mación para la participación, con énfasis en adaptación al cambio climático.

- Proyecto 821. Fortalecimiento de la gestión ambiental para la restauración, conservación, manejo y uso sostenible de los ecosistemas urbanos y de las áreas rurales.

- Proyecto 826. Control y Gestión ambiental a residuos peligrosos, orgánicos y escombros.

De lo anterior, se puede establecer que la mayoría de instrumentos corresponde a planes y que la gestión, propiamente dicha, no se plantea como tal, solamente se halla en función de un plan, como lo es el plan de gestión ambiental. Además, los proyectos, como la menor unidad operativa que permite llegar a la acción real, no muestran claridad y concreción en su contenido de gestión ambiental o socio ambiental colectivo.

En los proyectos, se encuentra que la gestión ambiental se entiende como una acción de educación ambiental, otras como gestión administrativa, otras como participación ambiental y otras como veeduría ambiental. Y aunque en la descripción inicial de los proyectos se nota un discurso más elaborado que el de los proyectos de las vigencias anteriores, ya en el detalle de las acciones para cumplir los objetivos específicos, se sigue recurriendo a las típicas de educación, de participación y de administración ambiental.

En cuanto a la manera de diseñar la planeación y la gestión ambiental, se observa el ingreso de los siguientes temas nuevos (a diferencia de los objetivos de calidad ambiental, de eco-eficiencia y de armonía, que se habían presentado desde 1992: "gestión del riesgo y de desastres", "socializa- ción y corresponsabilidad", "ordenamiento y gestión de la ciudad-región".

Es de anotar que en el plan de desarrollo Distrital, a diferencia del plan de gestión ambiental, el tema relativo a la gestión ambiental se separa de la gestión del riesgo, pero al mismo tiempo, se asume como una política que debe estar articulada con las demás políticas ambientales. Y respecto a los sistemas de información que se vienen elaborando, estos ayudan a mejorar la capacidad técnica para identificar y para comprender los conflictos ambientales, pero no se comprueba la forma en que ayudan a mejorar la coordinación de las acciones.

Al respecto, ċserá posible que los sistemas de gestión ambiental o de planeación puedan coordinar eficientemente acciones conjuntas y trasformadoras? La información es diferente de la gestión; la comunidad informada puede visualizar mejor su situación y hasta hacer prospectiva, sensibilizarse y generar conciencia, pero es la comunidad empoderada la que es capaz de vincular la información con la acción efectiva. La Web, por ejemplo, permite la generación de redes de contactos, pero muchos de ellos no pasan de ser virtuales y no es posible establecer aún cuáles son las redes que hay entretejidas virtualmente, para lograr acciones en el territorio real.

Diferentes aportes sobre la gestión ambiental colectiva: Para Moura (2008), el modelo de planeamiento estratégico adoptado por las ciudades capitalistas admite sólo la participación social, como mera formalidad; la población, en lugar de participar, es meramente "participada" en lo relativo a las decisiones tomadas por los especialistas en procesos que no 
incluyen a la comunidad. Los ciudadanos no son agentes activos en la producción del territorio, pero sí aparecen como receptores pasivos de los servicios ofertados.

Lo anterior, se presenta en el caso Bogotano y en gran parte del territorio colombiano, pues se ha utilizado el concepto, el método y los instrumentos de participación como un formalismo, lo cual, se puede comprobar únicamente con que cada uno de nosotros se pregunte: cen la elaboración de cuántos planes ambientales he participado? El asunto es que la participación real empieza en la misma elaboración participativa del instrumento plan, de lo contrario, seguimos produciendo instrumentos sin dolientes.

En la misma vía, Sáenz (2006) señala que una condición básica de sostenibilidad es la participación activa y el empoderamiento de ciudadanos desde la base, en los procesos, las actividades, los recursos, las proposiciones y las decisiones del desarrollo. Esta participación se da en procesos de concertación entre actores diversos hasta lograr construir un tejido social; por esto, la gestión de procesos de desarrollo es la generación de capacidades locales para lograr una mejor calidad de vida y no solamente un ordenamiento de la gestión de obras.

Al respecto, se observa que un asunto es armonizar los instrumentos de gestión ambiental de las autoridades ambientales nacionales, regionales y locales o armonizar los instrumentos de gestión del suelo; pero otra situación es lograr recuperar el tejido natural, con una participación efectiva de la sociedad, para lo cual, en primera instancia, se requiere que exista un tejido social local fuerte y, en este sentido, no existe en Bogotá un plan específico de gestión social, mucho menos, un proceso claro de gestión socio ambiental.

De manera contundente, Leff (2009) consideró que la gestión democrática del ambiente implica un proceso de concertación, que permita dirimir conflictos, pero que también abra opciones para crear diferentes estrategias de apropiación y de transformación de la naturaleza, dentro de los principios de racionalidad ambiental; la acción social es la que construye una nueva racionalidad ambiental.

En el caso de Bogotá, en teoría, los diferentes planes elaborados durante tres periodos de gobierno por las distintas secretarías técnicas del Distrito, tienen buenos planteamientos, destacando los del Jardín Botánico, quienes integran todo en la gestión ambiental territorial, pero entre las diferentes secretarías aún subsisten inconsistencias internas. Cuando se trata de la gestión, es mejor poca teoría y mucha efectividad, frente a mucha teoría y poca acción. Tal como sostiene Ostrom (2011), los recursos comunes no deben ni centralizarse, ni privatizarse.
De allí, que la articulación interinstitucional sea diferente a la articulación de los actores sociales claves, es decir, los usuarios directos, aquellos a los que no se les paga por hacer algo, sino que pagan impuestos para que alguien los oriente sobre cómo interactuar mejor con su territorio. La forma, el cómo es la acción, la acción colectiva que es la verdadera gestión ambiental. Al respecto, Posada (2009) manifiesta que es necesario identificar los distintos intereses que se pueden presentar respecto a un recurso natural o a una zona determinada y sus posibles tendencias, para luego establecer las estrategias que permitan una gestión socio ambiental acertada.

De forma similar, Andrade (2009) sostiene que son tan importantes los sujetos involucrados, beneficiarios o afectados, como los objetos de conservación que han recibido atención y la forma como se construye la naturaleza protegida, como una decisión de la sociedad, por ello, el reto ecosistémico es la construcción social de la nueva naturaleza protegida.

Pero estas posiciones tan enriquecedoras, no se ven plasmadas en el ejercicio de gestión ambiental de Bogotá; en parte, se encuentran en la teoría de los diferentes planes, tal como se aprecia en siguiente la aseveración de la AMB (2010): "hoy se comprende a Bogotá en el marco ambiental y cultural de territorios por cuencas, cerros, redes de humedales, páramos y ruralidad, porque los actores comunitarios y públicos requieren de un contexto mayor de incidencia y construcción...”. ¿¿uién?, ¿̇qué personas son las que tienen esta importante comprensión ambiental del territorio? Tal vez sea un objetivo de futuro (y de muy largo plazo), pero por ahora, esta racionalidad ambiental no se da en toda su dimensión y menos en coherencia con las necesidades socio ambientales de Bogotá.

La gestión territorial permite el avance del desarrollo local si es tratado como un proceso que involucra el surgimiento, las motivaciones, las actividades y los logros de los diferentes actores y agregan que, la cuestión ambiental apenas aparece en forma incipiente en estos procesos, pero que van en crecimiento unidos a una demanda por parte de los habitantes del territorio, en relación a las formas de producción imperantes (Aradas et al. 2012).

En esta misma vía, Fernández (2000) asume que el pensamiento ambiental contribuye en su desarrollo conceptual y metodológico, a analizar las posibilidades sistémicas de la modelación de los procesos del desarrollo territorial, permitiendo esclarecer los aspectos inherentes a la complejidad e interactividad de tales procesos, abriendo vías para aprender la interacción entre asentamiento y territorio. Este tratamiento sistémico de los procesos territoriales es un instrumento alternativo, que permite una mayor transparencia y da posibilidad de control social o comunitario. 
Bogotá tiene una responsabilidad mayor y lo ha asumido con instrumentos válidos, pero los procesos de recuperación ambiental exigen algo más que planes de instituciones públicas y, por ello, los compromisos ambientales públicos no pueden tener respuestas totales en periodos de gobierno de cuatro años. Se haría mal, si no asumimos una posición positiva y esperanzadora desde la academia, por ello, es importante considerar las reflexiones de autores, como Wong (2010), quien sugiere, por un lado, repensar las bases conceptual-filosóficas de la cuestión territorial y, por otro, flexibilizar las formas de instrumentación y de gestión de los procesos. Aquí, adquieren relevancia las nuevas tendencias de participación y de gestión de los actores sociales en los procesos de desarrollo, quienes se encuentran ganando espacios, más allá de ser representantes de comunidades.

Posibilidades que enmarcan a Bogotá en una ventaja comparativa sobre gestión ambiental: Una forma de analizar la eficiencia de los planes tienen que ver con la disponibilidad y el gasto presupuestal, que se ha previsto para temas ambientales en el territorio bogotano. Al respecto, según la AMB (2012), en el caso de las localidades, se invertirá en la solución a situaciones ambientales conflictivas de los territorios, según los proyectos de los planes de desarrollo local.

Como se observa en la figura 2, la comparación entre los seis principales municipios de Colombia, permiten ver a Bogotá no como el más extenso (es mayor el espacio territorial de Santa Marta), pero sí como la entidad territorial con mayor número de habitantes y con un presupuesto financiero notoriamente superior, respecto a los demás. En síntesis, se triplica la población (respecto al municipio que está por debajo), pero también se triplica el presupuesto disponible para la acción.

Entrando ahora en el detalle de la inversión presupuestada para temas territoriales y ambientales, se observa en la figura 3 , que es Bogotá el que mayor porcentaje de su presupuesto ha destinado en el actual periodo para inversión en temas territoriales y ambientales, con un $33 \%$ del total, con lo cual, se deduce que, en términos financieros, sí hay con qué hacer algo. Entonces, ¿dónde está el vacío?, ¿̇por qué siguen prevaleciendo tantos conflictos ambientales?

La respuesta está en el proceso de gestión ambiental territorial. El proceso, como un proceso sistémico, apenas está empezando. Si bien es destacable que exista un plan de gestión ambiental de largo plazo, que cubre hasta el 2038, las inquietudes contemporáneas surgen respecto a las formas concretas de vincular a la población eficientemente y con continuidad a sus causas, problemas y soluciones comunes.

Para Sáenz (2006), la gestión incluye el manejo participativo de programación de la inversión efectiva y manejo de los recursos en función del plan, monitoreo de procesos, evaluación, negociación, manejo de conflictos y rendición de cuentas; considera que este concepto es más amplio que el de administración o implementación de un plan, pues consiste en un proceso dinámico, que involucra a varios actores locales y los compromete a una actitud positiva que permita cambiar la situación.

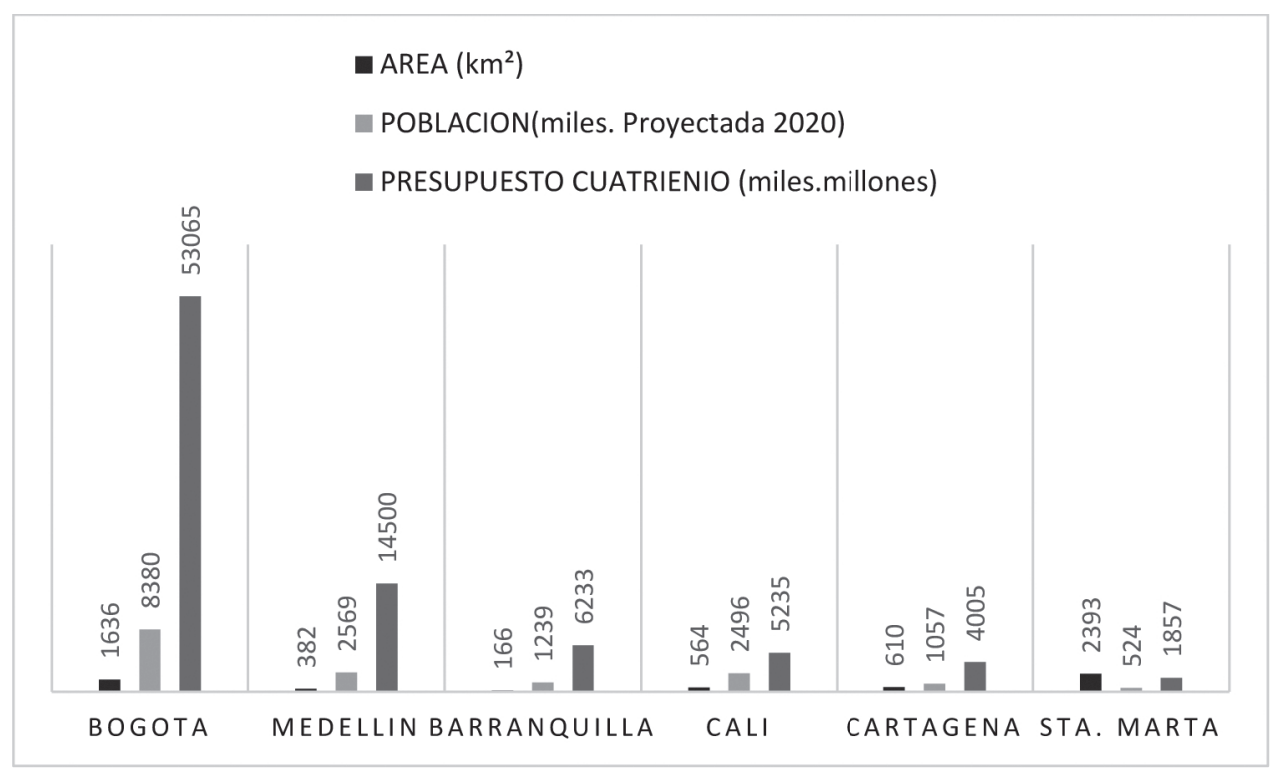

Figura 2. Comparación cifras básicas principales municipios de Colombia. 


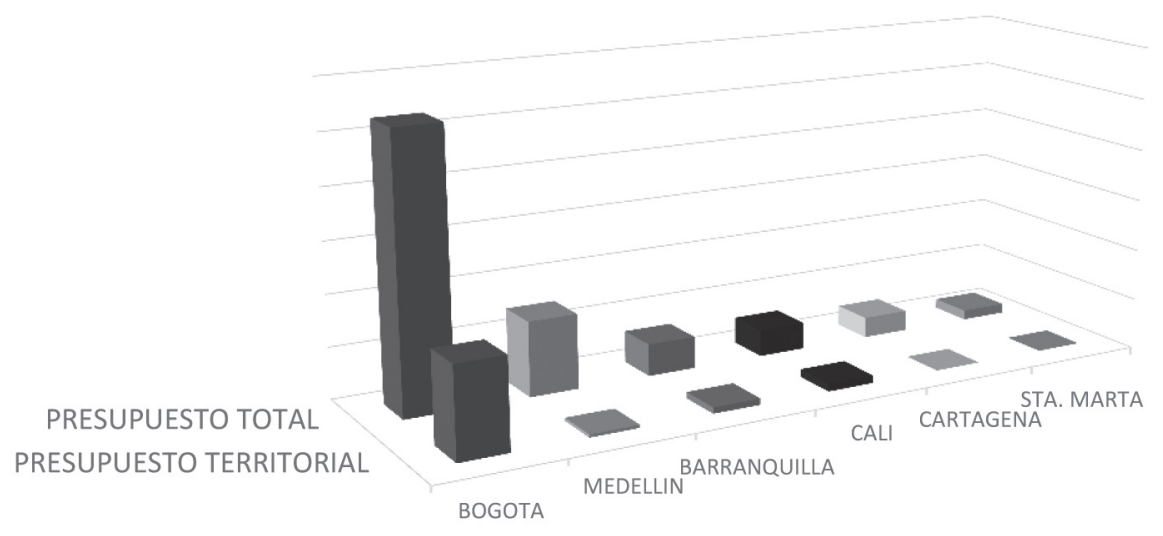

Figura 3. Comparación presupuestos y detalles presupuestales principales municipios de Colombia.

En Colombia, el Ministerio de Medio Ambiente -MMA- (1998) ya lo ha señalado en su política, pues advierte que, dada la complejidad del proceso, en razón de la diversidad de intereses involucrados y de las dificultades de orden técnico, político y económico, el ordenamiento ambiental del territorio debe adoptar un carácter gradual.

Según Posada (2012), para hacer realidad una transformación ambiental en un territorio es necesario que los grupos o el colectivo tengan intereses de hacerlo. Indefectiblemente, los intereses colectivos son intereses difusos, pero no por ello menos importantes que los intereses individuales reales. Cada grupo de interés tiene un aporte que debe ser considerado al momento de realizar una gestión socio ambiental o cualquier proyecto ambiental, que implique al colectivo social.

Se concluye, entonces, que en el caso de Bogotá existen muchas necesidades ambientales, pero también varias personas que podría hacer parte de la solución y un buen presupuesto para jalonar acciones concretas, pero que se requiere un ajuste estructural, que no parta tan sólo de los planes, sino de alternativas integradoras, diferentes y creativas, que bien pueden provenir de la misma sociedad.

También, se constata que se ha venido trabajando por separado el tema ambiental, la planeación, la gestión y el territorio; sin embargo, ya existen algunas posiciones que manifiestan la necesidad de integración de las partes, en relación con el todo, como un sistema. Es claro que, a nivel mundial, estamos en la era de las fusiones y requerimos pasar de la planeación individual a la acción colectiva, para poder estar en el contexto de la realidad que tenemos y que necesitamos. La gestión ambiental colectiva puede ser una buena respuesta para dinamizar los procesos de armonización del territorio no como un instrumento más, sino como acciones conjuntas, acciones transformadoras graduales en un horizonte de largo plazo.

Este horizonte ya ha tenido unos puntos de partida relevante en Colombia y, en especial, en Bogotá, si consideramos las visiones plasmadas en los documentos de política, como la agenda siglo XXI o Colombia 2020 o el mismo plan de gestión ambiental de Bogotá, proyectado al 2038; sin embargo, haciendo un simple ejercicio aritmético, se puede deducir que, tal vez, la efectividad de las acciones en el largo plazo se puedan medir y ver reflejadas en una realidad en, aproximadamente, en el 2050.

Para determinar esta temporalidad, se considera que llevamos más de 20 años desde la Constitución Política de Colombia haciendo planes de mejoramiento ambiental; que estamos empezando a teorizar sobre la gestión ambiental colectiva; que pasaremos por otra década para poder dinamizar la práctica del cuidado del ambiente (de forma momentánea, parcial e individual) y que necesitaremos de otras dos décadas para garantizar una práctica del cuidado del ambiente, de forma integral, constante y grupal, con lo cual, podremos ver materializar la teoría en hechos transformadores.

Agradecimiento: La autora agradece a la Universidad de Ciencias Aplicadas y Ambientales U.D.C.A, por financiar el proyecto de investigación "Gestión Ambiental Territorial en Bogotá", que dio origen a este manuscrito. Conflicto de Intereses: El manuscrito fue preparado y revisado por la autora, quien declara ser la única autora y que no presenta conflicto de intereses. 


\section{BIBLIOGRAFÍA}

1. ALCALDÍA MAYOR DE BOGOTÁ -AMB-. 2012. Plan de desarrollo económico y social y de obras públicas para Bogotá distrito capital 2012 - 2016: Bogotá humana. Colombia. 48p.

2. ALCALDÍA MAYOR DE BOGOTÁ -AMB-. 2009. Plan de Gestión para el Desarrollo Rural Sostenible, del Distrito de Bogotá. Secretaria Distrital de Ambiente. Secretaria Distrital de Planeación. Colombia. 170p.

3. ALCALDÍA MAYOR DE BOGOTÁ -AMB-. 2010. Jardín Botánico José Celestino Mutis. Territorios ambientales y gestión ambiental territorial en Bogotá. Colombia. 18p.

4. ALCALDÍA MAYOR DE BOGOTÁ -AMB-. 2008. Secretaria Distrital de Ambiente. Plan de gestión ambiental del distrito capital 2008-2038. Decreto Distrital 456 de 2008. Colombia. 72p.

5. ANDRADE, G. 2009. ¿El fin de la frontera? Reflexiones desde el caso colombiano para una nueva construcción social de la naturaleza protegida. Rev. Est. Soc. Colombia.32:48-59.

6. ARADAS, M.; DURE, L.; PAGANI, V.; CARUSO, C.; PERA PUJOL, P. 2012. Convergencias y Divergencias en Experiencias de Gestión Local en áreas rurales de Argentina. Rev. Econ. Agr. (Argentina).16:1-19.

7. DEPARTAMENTO NACIONAL DE PLANEACIÓN -DNP. 2013. Informe de ejecuciones presupuestales del año anterior 2012. Disponible desde Internet en: www.dnp.gov.co (con acceso 01/02/2014).

8. FERNÁNDEZ, R. 2.000. Gestión ambiental de ciudades. Teoría crítica y aportes metodológicos. 1a edición. Serie Textos Básicos para la Formación Ambiental PNUMA. Chile. 335p.

9. LEFF, E. 2009. Racionalidad ambiental la reapropiación social de la naturaleza. Siglo XXI editores. Segunda edición. México. 509p.

10. MINISTERIO DE AMBIENTE, VIVIENDA Y DESARROLLO TERRITORIAL -MAVDT-. 2008. Política de Gestión ambiental Urbana. Colombia. 54p.
11. MINISTERIO DEL MEDIO AMBIENTE -MMA-. 1998. Oficina asesora de ordenamiento ambiental. Lineamientos para la política nacional de ordenamiento ambiental del territorio. Colombia. 68p.

12. MOURA, R. 2008. Coerción en las prácticas urbanas y fragmentación de la cohesión social: una mirada desde las ciudades brasileñas. Revista papeles (Brasil).101:89-99.

13. OSTROM, E. 2011. El Gobierno de Los Bienes Comunes: La Evolución de Las Instituciones de Acción Colectiva. Fondo de cultura económica México. $2^{\mathrm{a}}$ ed. 403p.

14. POSADA, A. 2009. Intereses ambientales: reflexiones sobre la aplicación de lo difuso. Rev. Ingenierías. (Colombia). 8(14):11-20.

15. POSADA, A. 2012. Análisis de intereses ambientales mediante lógica difusa: caso reserva forestal regional del norte de Bogotá-Colombia. Rev. U.D.C.A Act. \& Div. Cient. (Colombia). 15(2):437-498.

16. QUIJANO, H. 2012. Descentralización y medio ambiente en Colombia. Fundación Konrad Adenauer. Colombia. $1^{\text {a }}$ ed. 149 p.

17. REPÚBLICA DE COLOMBIA. 1991. Constitución política de Colombia. 139p.

18. RETOLOZA, I. 2010. Teoría de cambio, un enfoque de pensamiento - acción para navegar en la complejidad de los procesos de cambio social. PNUD/Hivos. Guatemala. 49p.

19. SÁENZ, A. 2006. Estándares para la medición del desarrollo local. Cuadernos EBAPE. (Brasil). 4(4):1-31.

20. VELÁSQUEZ, F. 2010. La planeación territorial en Colombia: contexto, trayectoria y experiencias. Cuadernos descentralistas \#25. Perú. $1^{\text {a }}$ ed. 196p.

21. WONG, P. 2010. Ordenamiento ecológico y ordenamiento territorial: retos para la gestión del desarrollo regional sustentable en el siglo XXI. Rev. (México). Estudios Sociales (número especial):11-39.

Recibido: Febrero 24 de 2014

Aceptado: Abril 25 de 2014

\section{Como citar:}

Posada, A. 2014. De la planeación a la gestión ambiental colectiva: posibilidades en Bogotá-Colombia. Rev. U.D.C.A Act \& Div. Cient. 17(1): 257-264. 